\title{
Model Reference Adaptive Control on the Output of the Power Generation System
}

\author{
Lixin $\mathrm{Ma}^{1, \mathrm{a}}$, Chun $\mathrm{Liu}^{2, \mathrm{~b}}$ \\ ${ }^{1}$ School of Optical-Electrical and Computer Engineering, Dept. of Electrical Engineering, Univ. of \\ Shanghai for Sci.\& Tech, Shanghai 200093 China \\ ${ }^{2}$ School of Optical-Electrical and Computer Engineering, Dept. of Electrical Engineering, Univ. of \\ Shanghai for Sci.\& Tech, Shanghai 200093 China

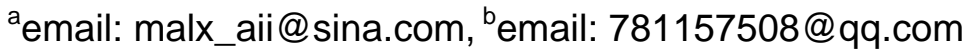

\begin{abstract}
Keywords: Power Generation Systems Output; Intelligent Control; Model Reference Adaptive Control System(MRACS); Gradient Descent;
\end{abstract}

\begin{abstract}
The stability of the output of power generation systems plays an important role in the production efficiency of the power grid. As the research on control method progresses, new theory appeared, and the output control has greatly improved. Boiler steam temperature control process related to how much output, but it has many disturbance signal, furthermore the control is very complex. In this paper, adopting the model reference adaptive control system to control the steam temperature, it can quickly track the changes in the output of power generation system, therefore increase the system stability and robust to disturbance. And simulation experiment results show that the model reference adaptive control system can get better dynamic performance parameters and better output than the traditional control method. which demonstrates certain theoretical guidance and as well as field application prospect.
\end{abstract}

\section{Introduction}

In the development of domestic electric power industry, large capacity thermal power unit has become the mainstream of the electric power industry. At present, the traditional PID control [2] has been widely applied in output control of the power systems in power plants, but for the main steam temperature control that has many disturbance factors is difficult to be controlled, where it is difficult to establish accurate mathematical model adopting traditional PID control method. Therefore, it has become the top priority to apply advanced intelligent control instead of traditional process control in power plants. The above situation puts forward a model reference adaptive control system [7]. It can restore the stability quickly, and reduce the error. Its development process described in the literature [1].

\section{Summary of Steam Temperature Control System}

As shown in Fig 1, due to the temperature of superheater entrance determines the outlet temperature, hence it controlled through mixing water to the superheater entrance. Finally the value of steam temperature in superheater export is controlled within the prescribed scope. The steam is sent to the reheater heat again after the steam to do work in high-pressure cylinder, then as reheat steam into the medium pressure cylinder. The conventional PID[6] control is used in the process.

But in the running process, there are still problems in the control process. Adjusting the recirculation of combustion gases can control the reheat steam temperature, but the main steam temperature may also be affected; furthermore, if the steam temperature is changed, the heat exchange will be affected The conventional control methods can not deal with these problems. 


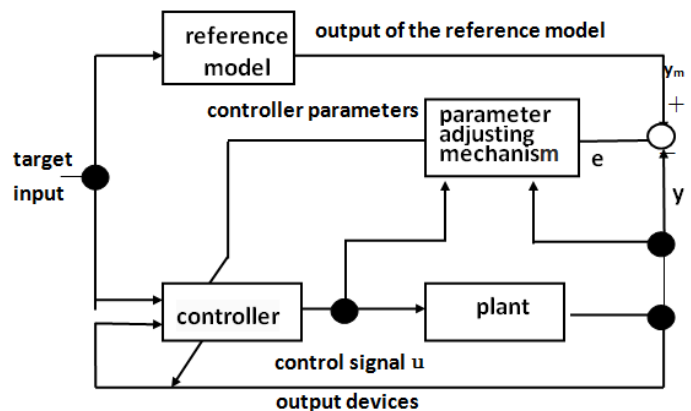

Fig.1. System of the steam temperature

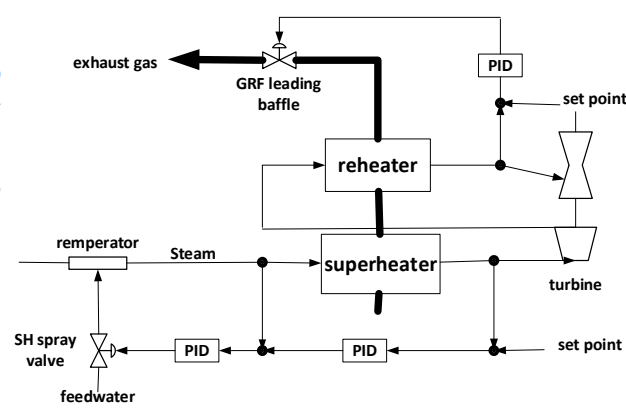

Fig.2. Structure diagram of the MRACS

\section{Principle of Model Reference Adaptive Control System}

In order to solve above problems, the paper puts forward the model reference adaptive control system(MRACS). It can make the dynamic characteristic of the reference model to be traced by plant, and regulate the main steam temperature change when the multiple disturbance factors appearing, which makes the error tend to be stable quickly, and gets the satisfactory control performance.

The reference model of MRACS is established in order to make the output of the plant to achieve the desired state. As shown in Fig 2, giving the reference model and the controlled device to join the same signal, the expectation is given by output state of the reference model. Based on the above description, the basic principle of MRACS is: the parameter adjustment mechanism of system adjusts parameters of the controlled device by adjusting itself parameters, eventually reduce the error between the controlled device output and the reference model output. The controller parameters are obtained by certain algorithm after the parameters adjustment mechanism obtains the $\mathrm{y}_{\mathrm{m}}, \mathrm{y}$, e and other state variables. When system convergence, the operation performance of the plant is the same as the reference model's, in order to achieve the reference model is tracked.

\section{The Design of Parameter Adjusting Mechanism}

The parameter adjustment mechanism of MRACS can be obtained by many different methods, such as Gradient method, Popov hyper stability theory, Lyapunov stability theory, regardless of method is simple or complex, the result is the same. This paper will adopt the Gradient method [3-5] to calculate the parameter adjustment mechanism.

Selecting a variable gain $\mathrm{K}_{\mathrm{C}}$ as the controller parameter. The actual output of the controlled device is defined as $\mathrm{y}$; the output of reference model is defined as $\mathrm{y}_{\mathrm{m}}$; the generalized error between the controlled device actual output and the reference model output is defined as e. The transfer function of reference model is expressed as

$$
G_{m}=\frac{K N(\mathrm{~s})}{D(\mathrm{~s})} \text {. }
$$

\section{(1)}

The transfer function of the controlled device is expressed as $\quad G=\frac{K_{C} N(\mathrm{~s})}{D(\mathrm{~s})}$.

The generalized error is expressed as

$$
\begin{aligned}
& \mathrm{e}=y_{m}-y . \\
& I P_{R U}=\frac{1}{2} \int_{t_{0}}^{t} e^{2} d \tau .
\end{aligned}
$$

Assume the performance indicators is

The next set up the parameter adjustment mechanism. In order to the performance index of IPRM can get the minimum value, setting the adjustable gain $\mathrm{K}_{\mathrm{C}}$ is constant. Assume that the adjustable gain $\mathrm{K}_{\mathrm{C}}$ can be expressed as

$$
K_{C}=\Delta K_{C}+K_{c 0} \text {. }
$$

Adopting the Gradient method to calculate parameter adjustment mechanism. The search process performs in a hyper surface that constituted by the performance index of IPRM. Assumes the A as the starting point, the curved round as the track, its performance index is IPRM1, then along the negative gradient direction search until find the first point that the performance index is IPRM2, and then search for the second point until the point take the minimum value. 
Any plane gradient as follow

$$
\begin{aligned}
& \operatorname{gradIP_{RИ}}=\frac{\partial I P_{R И}}{\partial K_{C}} . \\
& \operatorname{gradII} P_{R И}=\int_{t_{0}}^{t} e(\tau) \frac{\partial e(\tau)}{\partial K_{C}} d \tau .
\end{aligned}
$$

Incorporating equations(4)and(6) becomes

$\lambda$ is defined the search step length, then along the negative gradient direction search one step. The variation of $\mathrm{K}_{\mathrm{C}}$ as

$$
\Delta K_{C}=-\lambda \operatorname{grad} I P_{R \|} .
$$

Substituting (7) and (8)into (5) yields

$$
K_{C}=-\lambda \operatorname{gradIP} P_{R U}+K_{C 0}=-\lambda \int_{t_{0}}^{t} e(\tau) \frac{\partial e(\tau)}{\partial K_{C}} d \tau+K_{C 0}
$$

Substituting (3) into (9) yields

$$
K_{C}=-\lambda e \frac{\partial e(\mathrm{t})}{\partial K_{C}}=\lambda e \frac{\partial y}{\partial K_{C}} .
$$

The equations(10) is the parameter adjustment structure of adjusting $K_{C}$. Where, əy/əK $K_{C}$ is called sensitivity function

\section{Simulation Research and Analysis}

According to above theory research, the feasibility of the theory is proved by simulation in Matlab. The superheater dynamic characteristics of the steam temperature control system under the load of $30 \%$ of $300 \mathrm{mw}$ boiler as table.1, selecting the first-order system as the transfer function of the controlled device and the transfer function as table.2:
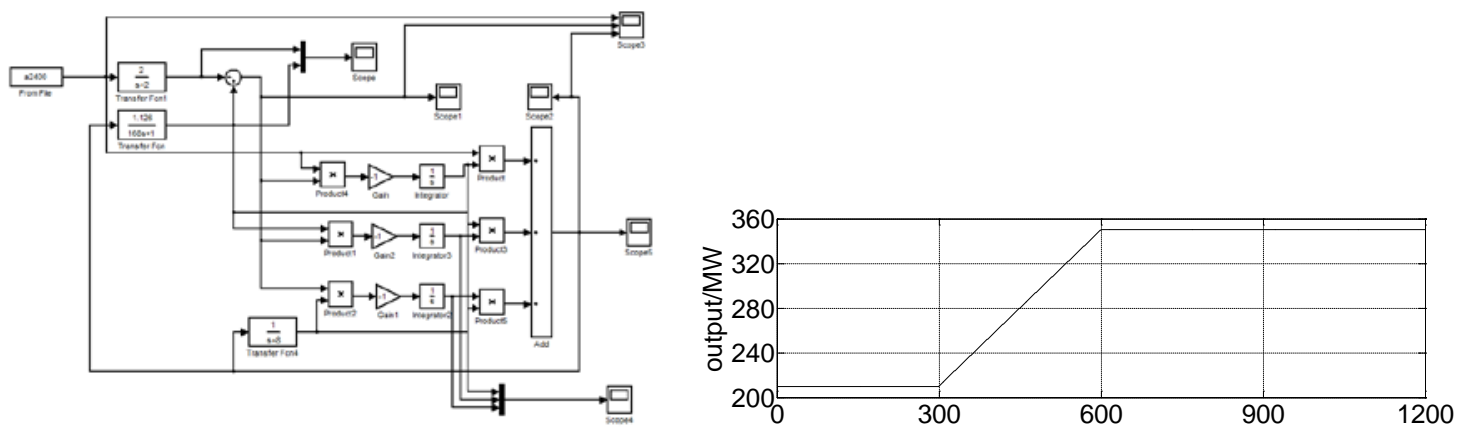

Fig.3. the structure of the MRACS simulation system

Fig. 4. Input curve of $1200 \mathrm{~s}$ load

Table.1. The superheater dynamic characteristics

Table.2. The transfer function

\begin{tabular}{cccc}
\hline guide front zone & Inert zone & controlled device & reference model \\
\hline$W_{1}=\frac{6.506}{(26 \mathrm{~s}+1)^{2}}$ & $W_{2}=\frac{1.568}{(48.8 \mathrm{~s}+1)^{2}}$ & $G(\mathrm{~s})=\frac{1.126}{168 \mathrm{~s}+1}$ & $G_{m}(\mathrm{~s})=\frac{2}{\mathrm{~s}+2}$ \\
\hline
\end{tabular}

The data of the load output as the input plant ,the variation of load range is $210 \mathrm{mw}-350 \mathrm{mw}$. Make a comparision with the MRACS method and the traditional control method, the result is shown in fig 4-6.

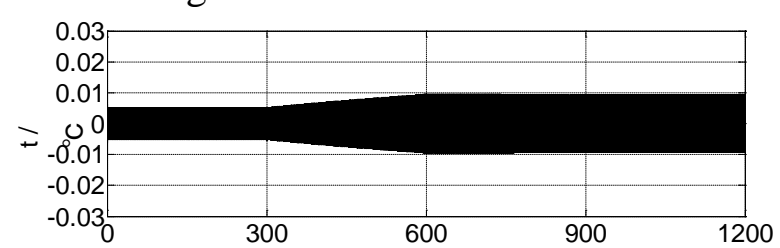

Fig.5. Error curve of $1200 \mathrm{~s}$ MRACS

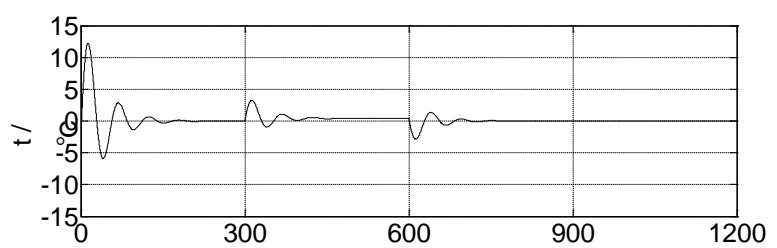

Fig.6. Error curve of 1200 s traditional PID

From the above figure we can see:

(1)From fig.4 and fig.5 show that the error change of the MRACS follow the change of the load. Although fig.5 system has some fluctuations, the error is still smooth changes in the area of near zero, the restore stability time is less, and it has the satisfactory performance .

(2) From fig.5 and fig.6 show that the output error of the MRACS is far less than the error of traditional PID control, the error range of the former is around 0.01 and the reaction time is short, while the error range of the latter is around 5, thus the control effect of the MRACS is very 
significant for power generating system.

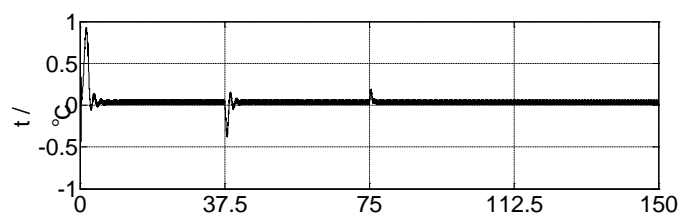

Fig.7. Error curve line of 150 s MRACS

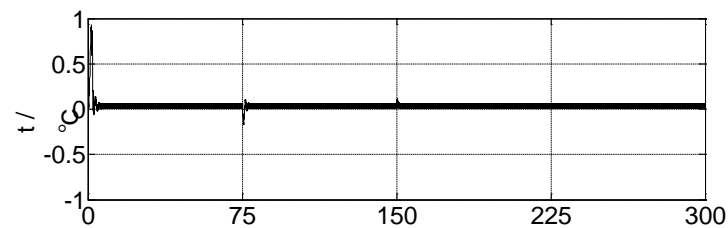

Fig.8. Error curve line of 300 s MRACS

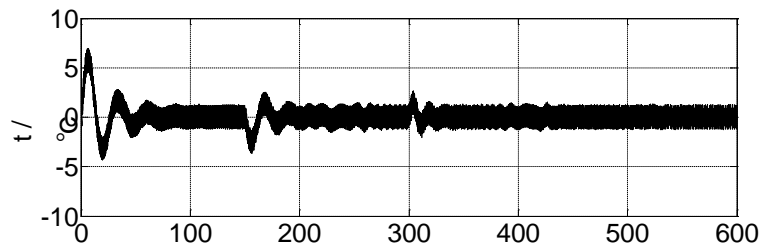

Fig.9. Error curve line of $600 \mathrm{~s}$ MRACS

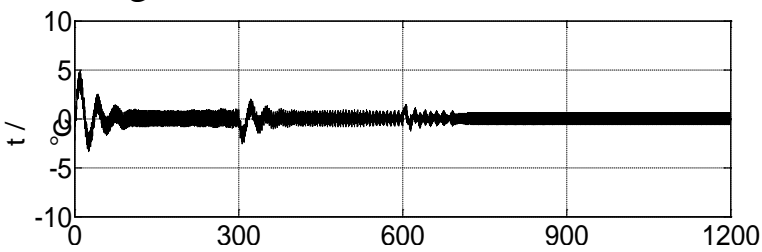

Fig.10. Error curve line of 1200 s MRACS

Although the simulation results show that the performance of MRACS is better than the performance of the traditional PID control, the oscillation is obvious in the process of the regulating. Hence, giving the controller to join the low pass filter, so that it can weaken the oscillation effect. Due to the adjust speed of system is very quickly ,the simulation is divided into 4 stages. There are $150 \mathrm{~s}, 300 \mathrm{~s} 600 \mathrm{~s}$, and $1200 \mathrm{~s}$ respectively, and then comparing their simulations results .

The simulation results as shown in fig.7 and fig.8, the low-pass filter have not be joined in system, compared with fig.5, the dynamic adjusting was obvious when output changed. But at $150 \mathrm{~S}$ in Fig.7 can be seen that the dynamic process has not obvious. The same result appears in fig.8. The simulation results as shown in fig.9 and fig.10 after join the filter the filter cut-off frequency is 0.01Hz. Comparing Fig.9, Fig.10 and Fig.7, fig.8 can be seen that the dynamic adjustment process is significant when output change, so enhance the performance of the system. But the error increases, the study about the system control will continue.

\section{Conclusion}

This paper focused on the main steam temperature control, adopts the MRACS method to study the controlled device. The simulation results proved that the new control method can get better dynamic performance parameters and achieved better control output, which MRACS can effectively improve power generation equipment in the temperature control problems caused by multiple disturbance load fluctuations. Oscillation problem is improved, the effect is good, but it still need further study to reduce error and oscillation at the same time.

\section{References:}

[1] X.M. Xie, F. Ding, Adaptive Control System. Beijing: Tsinghua University Press.2002.

[2] G.Y. Wang, P. Han and D.F. Wang, Studies And Application of PFC-PID Cascade Control Stragety in Main Steam Temperature Control System , Proceedings of the CSEE, vol.22, no. 12, 2002, pp. 50-55.

[3] S. Matsumura and K. Hirayama: Control Technology in Power Generating Equipment. Beijing: China Machine Industry Press.2011.

[4] Q. Fang, L.P. Chen, in: Model Reference Adaptive Control System Design of Localizer, Journal of Zhejiang University(Engineering and Technology Edition), vol.47, no. 12, 2013,pp.2235-2242. [5] X.Y. Liu, L.L. Yao and Q.Z. Li, Application of Model Reference Adaptive Control Strategy in Grid Connected PV System, Power System, vol.33, no.9, 2013, pp. 32-37

[6] H. Shi, Application of LPMSM Model-based Adaptive Control System, Control Engineering of China, vol.15, no.5, 2008, pp.183-185. 